\title{
QND measurements and state preparation in quantum gases by light detection
}

\author{
Igor B. Mekhov ${ }^{1,2, \text { * } \text { and Helmut Ritsch }}{ }^{1}$ \\ ${ }^{1}$ Institut für Theoretische Physik, Universität Innsbruck, Innsbruck, Austria \\ ${ }^{2}$ St. Petersburg State University, Faculty of Physics, St. Petersburg, Russia
}

\begin{abstract}
We consider light scattering from ultracold quantum gas in optical lattices into a cavity. The measurement of photons leaking out the cavity enables a quantum nondemolition (QND) access to various atomic variables. The time resolved light detection projects the motional state to various atom-number squeezed and macroscopic superposition states that strongly depend on the geometry. Modifications of the atomic and light properties at a single quantum trajectory are demonstrated. The quantum structure of final states can be revealed by
\end{abstract}

PACS numbers: 03.75.Lm, 42.50.-p, 05.30.Jp, 32.80.Pj

Quantum gases in optical lattices are of fundamental interest, as they provide an excellent testbed to study multipartite entanglement and many-body states, useful in condensed matter and quantum information [1]. Usually, the role of light is reduced to a classical auxiliary tool for creating intriguing atomic states. In contrast, here we consider an ultimate quantum level, where quantum natures of both matter and light play a key role. This emerging level joining quantum optics [especially cavity quantum electrodynamics (QED)] and quantum gases, only recently became achievable and stimulated novel experimental [2] and theoretical studies [3, 4, , 5].

We show that the atom-light entanglement enriches physics and enables the quantum nondemolition (QND) measurement and manipulation of atomic states. Observing light allows to prepare different types of atom number squeezed and macroscopic superposition states. Note that the type of many-body states depends on the optical geometry. The quantum structure of final states can be revealed by further observations of the same sample, which is an advantage over destructive schemes [1, [] $]$.

As we consider off-resonant interaction, independent of a particle level structure, our model might be also applied to other phenomena in molecular physics [7], where the molecule number fluctuations are important, and solid-state systems as semiconductors [Bose-Einstein condensates (BEC) of exciton-polaritons] [8] and superconductors 9] (circuit cavity QED). Besides, the squeezed and macroscopic superposition states find applications in quantum interferometry and metrology [10].

Model. We consider (cf. Fig. (1) $N$ ultracold atoms in an optical lattice of $M$ sites formed by strong off-resonant laser beams. A region of $K \leq M$ sites is also illuminated by a weak probe, which is scattered into a cavity. We will investigate, how the measurement of photons leaking the cavity will affect the atomic quantum state.

The theory is based on the generalized Bose-Hubbard model taking into account the light quantization [3, 5]. In contrast to Ref. [5], we assume dynamics and measurement of the cavity mode is faster than atomic tunneling $[3,4]$. Thus, neglecting the influence of tunneling

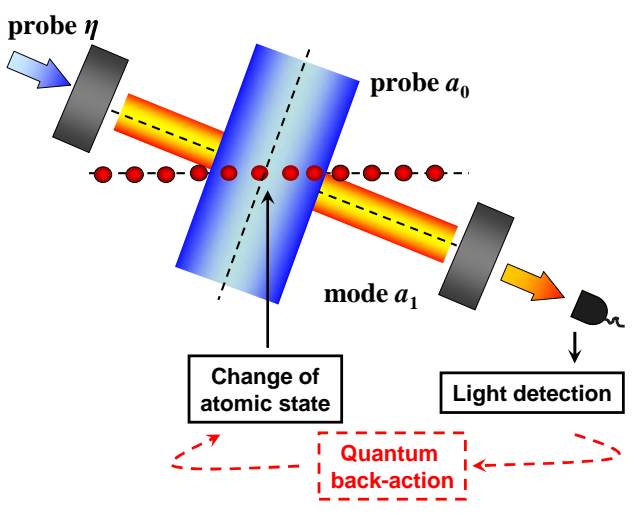

FIG. 1: (Color online) Setup. A lattice is illuminated by the transverse probe $a_{0}$ and probe through a mirror $\eta$. The photodetector measures photons leaking the cavity. Due to the quantum back-action, the light measurement leads to the modification of the atomic quantum state.

on light, we get the effective many-body Hamiltonian:

$$
\begin{array}{r}
H=\hbar\left(\omega_{1}+U_{11} \hat{D}_{11}\right) a_{1}^{\dagger} a_{1}+\hbar U_{10}\left(\hat{D}_{10}^{*} a_{0}^{*} a_{1}+\hat{D}_{10} a_{0} a_{1}^{\dagger}\right) \\
-i \hbar\left(\eta^{*} a_{1}-\eta a_{1}^{\dagger}\right),(1)
\end{array}
$$

where $a_{1}$ is the cavity-mode annihilation operator and $a_{0}$ is the c-number probe amplitude of the frequencies $\omega_{1, p}$ and spatial mode functions $u_{1,0}(\mathbf{r}) . U_{l m}=g_{l} g_{m} / \Delta_{a}$ $(l, m=0,1)$, where $g_{1,0}$ are the atom-light coupling constants, $\Delta_{a}=\omega_{1}-\omega_{a}$ is the cavity-atom detuning, $\eta$ is the probe through a mirror at $\omega_{p}$. We assumed the probecavity detuning $\Delta_{p}=\omega_{p}-\omega_{1} \ll \Delta_{a}$. The operators $\hat{D}_{l m}=\sum_{j=1}^{K} u_{l}^{*}\left(\mathbf{r}_{j}\right) u_{m}\left(\mathbf{r}_{j}\right) \hat{n}_{j}$ sum contributions from all illuminated sites with the atom-number operators $\hat{n}_{j}$ at the position $\mathbf{r}_{j}$. Here, the rotating-wave approximation and adiabatic elimination of the exited state were used.

The first term in Eq. (1) describes the atom-induced shift of the cavity resonance. The second one reflects scattering (diffraction) of the probe into a cavity. For a quantum gas the frequency shift and probe-cavity coupling coefficient are operators, which leads to different light scattering from various atomic quantum states [3].

The Hamiltonian (1) describes QND measurements of 
variables related to $\hat{D}_{l m}$ measuring the photon number $a_{1}^{\dagger} a_{1}$ [11]. Note, that one has a QND access to various many-body variables, as $\hat{D}_{l m}$ strongly depend on the lattice and light geometry via $u_{0,1}(\mathbf{r})$. This is an advantage of the lattice comparing to single- or double-well setups, where the photon measurement back-action was considered [12]. Moreover, such a geometrical approach can be extended to other quantum arrays, e.g., ion strings [13].

For example, $\hat{D}_{11}$ can reduce to the operator $\hat{N}_{K}$ of atom number at $K$ sites [3]. If the probe and cavity modes are coupled at a diffraction maximum (Bragg angle), i.e., all atoms scatter light in phase, the probe-cavity coupling is maximized, $\hat{D}_{10}=\hat{N}_{K}$. If they are coupled at a diffraction minimum, i.e., neighboring atoms scatter out of phase, $\hat{D}_{10}=\sum_{j=1}^{K}(-1)^{j+1} \hat{n}_{j}$ is the operator of number difference between odd and even sites. Thus, the atom number as well as number difference can be nondestructively measured. Note, that those are just two of many examples of how a QND-variable, and thus the projected state, can be chosen by the geometry.

Measurement back-action. The expression for the initial motional state of atoms reads

$$
|\Psi(0)\rangle=\sum_{q} c_{q}^{0}\left|q_{1}, . ., q_{M}\right\rangle,
$$

which is a superposition of Fock states reflecting all possible classical configurations $q=\left\{q_{1}, . ., q_{M}\right\}$ of $N$ atoms at $M$ sites, where $q_{j}$ is the atom number at the site $j$. This superposition displays the uncertainty principle, stating that even a single atom can be delocalized in space.

While interacting, the light and atoms get entangled. Quantum mechanics predicts that measurements of one subsystems (light) provides conditional information about, or affects, another one (gas). We will show, how the atomic uncertainty is affected by the light detection.

We use the open system approach [14 for counting photons leaking the cavity of decay rate $\kappa$. When a photon is detected, the jump operator is applied to the state: $\left|\Psi_{c}(t)\right\rangle \rightarrow a_{1}\left|\Psi_{c}(t)\right\rangle$. Between the counts, the system evolves with a non-Hermitian Hamiltonian $H-i \hbar \kappa a_{1}^{\dagger} a_{1}$. Such an evolution gives a quantum trajectory for $\left|\Psi_{c}(t)\right\rangle$ conditioned on the detection of photons at times $t_{1}, t_{2}, \ldots$

It is known 15] that, if a coherent probe illuminates a classical atomic configuration in a cavity, the light state is proportional to a coherent state $\left|\alpha_{q}(t)\right\rangle$ with $\alpha_{q}(t)$ given by the classical Maxwell's equation. Thanks to the approximation, where the tunneling does not affect light, we can get a simple analytical solution of the coupled lightmatter dynamics. Each atomic Fock state in Eq. (2) will be correlated with a coherent light state with parameters given only by the corresponding configuration $q$ : $\left|\Psi_{c}(t)\right\rangle=\sum_{q} c_{q}^{0} \exp \left[\Phi_{q}(t)\right]\left|q_{1}, \ldots, q_{M}\right\rangle\left|\alpha_{q}(t)\right\rangle / F(t)$, where $F(t)$ gives the normalization. So, the problem to find $\left|\Psi_{c}(t)\right\rangle$ reduces to finding $\alpha_{q}(t), \Phi_{q}(t)$ for all classical configurations forming the initial $|\Psi(0)\rangle$. Although a solution is available for any $t$, we present it for $t>1 / \kappa$, when the steady state is achieved in all $\alpha_{q}(t)$, and assuming the first photon was detected at $t_{1}>1 / \kappa$.

Due to the steady state in all $\alpha_{q}(t)$, the solution is independent of the detection times and after $m$ counts is

$$
\begin{array}{r}
\left|\Psi_{c}(m, t)\right\rangle=\frac{1}{F(t)} \sum_{q} \alpha_{q}^{m} e^{\Phi_{q}(t)} c_{q}^{0}\left|q_{1}, \ldots, q_{M}\right\rangle\left|\alpha_{q}\right\rangle, \\
\alpha_{q}=\frac{\eta-i U_{10} a_{0} D_{10}^{q}}{i\left(U_{11} D_{11}^{q}-\Delta_{p}\right)+\kappa}, \\
\Phi_{q}(t)=-\left|\alpha_{q}\right|^{2} \kappa t+\left(\eta \alpha_{q}^{*}-i U_{10} a_{0} D_{10}^{q} \alpha_{q}^{*}-\text { c.c. }\right) t / 2,
\end{array}
$$

where $D_{l m}^{q}=\sum_{j=1}^{K} u_{l}^{*}\left(\mathbf{r}_{j}\right) u_{m}\left(\mathbf{r}_{j}\right) q_{j}$ is a realization of $\hat{D}_{l m}$ at $\left\{q_{1}, . . q_{M}\right\} ; a_{0}, \eta$, and $\alpha_{q}$ all oscillating in steady state at $\omega_{p}$ were replaced by their constant amplitudes.

As we see, each light amplitude $\alpha_{q}(t)$, Eq. (4), is given by a Lorentzian corresponding to classical optics. Eq. (3) shows that the probability to find an atom configuration $q, p_{q}(m, t)=\left|\alpha_{q}\right|^{2 m} \exp \left(-2\left|\alpha_{q}\right|^{2} \kappa t\right)\left|c_{q}^{0}\right|^{2} / F^{2}$, changes in time due to the photodetection. This demonstrates the back-action of the light measurement on the atomic state.

In the following, we will show consequences of Eq. (3) for two cases, where only one probe $\left(a_{0}\right.$ or $\left.\eta\right)$ exists. For transverse probing $\left(a_{0} \neq 0\right)$, we also neglect the mode shift, assuming $U_{11} D_{11}^{q} \ll \kappa$ or $\Delta_{p}$. Thus, in both examples, $\alpha_{q}$ (4) depends on the configuration $q$ only via a single statistical quantity now called $z: z=D_{11}^{q}$ for cavity probing $(\eta \neq 0)$, and $z=D_{10}^{q}$ for transverse probing.

From Eq. (3) we can determine the probability distribution of finding a given $z$ after time $t$ as

$$
p(z, m, t)=\left|\alpha_{z}\right|^{2 m} e^{-2\left|\alpha_{z}\right|^{2} \kappa t} p_{0}(z) / F^{2},
$$

where the initial distribution $p_{0}(z)=\sum_{q^{\prime}}\left|c_{q^{\prime}}^{0}\right|^{2}$, such that all configurations $q^{\prime}$ have the same $z ; F^{2}=$ $\sum_{z}\left|\alpha_{z}\right|^{2 m} \exp \left(-2\left|\alpha_{z}\right|^{2} \kappa t\right) p_{0}(z)$ provides normalization.

Transverse probing at diffraction maximum. As was mentioned, at the Bragg angle, $\hat{D}_{10}=\hat{N}_{K}$ is the operator of atom number at $K$ sites. So, $z$ varies from 0 to $N$ reflecting possibilities to find any atom number at $K$ sites. The light amplitudes (4) $\alpha_{z}=C z$ are proportional to $z, C=i U_{10} a_{0} /\left(i \Delta_{p}-\kappa\right)$. The probability (6) reads

$$
p(z, m, t)=z^{2 m} e^{-z^{2} \tau} p_{0}(z) / \tilde{F}^{2}
$$

with a characteristic time $\tau=2|C|^{2} \kappa t$.

When time progresses, both $m$ and $\tau$ increase with a probabilistic relation between them. The Quantum Monte Carlo method 14] establishes such a relation, thus giving a trajectory. Note, that thanks to the simple analytical solution (3), it gets extremely simple. In each step, it consists in the calculation of the photon number in the state given by Eq. (3) and comparing it with a random number generated in advance, thus, deciding whether the detection or no-count process has happened.

If the initial atom number $z$ at $K$ sites is uncertain, $p_{0}(z)$ is broad [for the superfluid (SF) it is nearly 

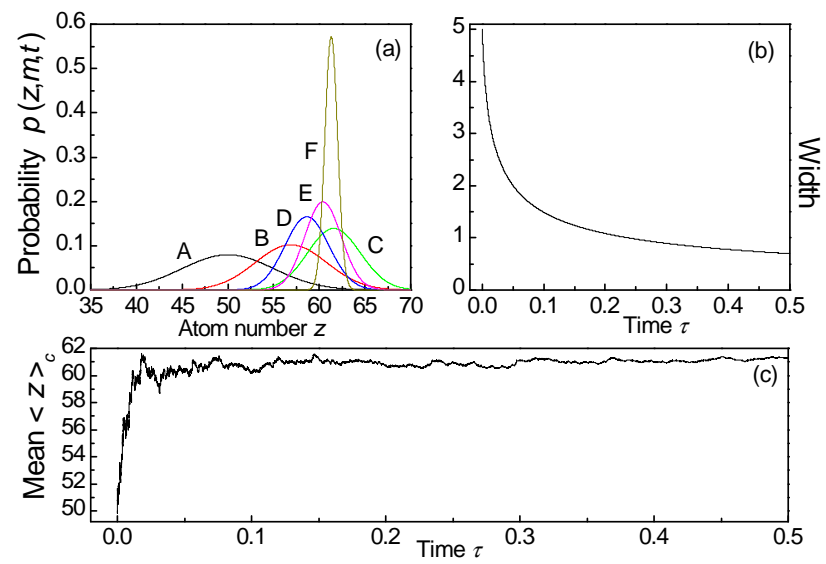

FIG. 2: (Color online) Photodetections at diffraction maximum. (a) Shrinking atom number distribution at different times $\tau=0,0.005,0.018,0.03,0.05,0.5$ (A-F); (b) decreasing width $\delta z ;(\mathrm{c})$ stabilizing mean atom number $\langle z\rangle_{c}$. Initial state: SF, $N=100$ atoms, $K=M / 2=50$ illuminated sites.

Gaussian [3]], and Eq. (7) shows that $p(z, m, t)$ is strongly modified during the measurement. The function $z^{2 m} \exp \left(-z^{2} \tau\right)$ has its maximum at $z_{1}=\sqrt{m / \tau}$ and full width at half maximum (FWHM) $\delta z \approx \sqrt{2 \ln 2 / \tau}$ (for $\left.\delta z \ll z_{1}\right)$. Thus, multiplying $p_{0}(z)$ by this function will shrink the distribution $p(z, m, t)$ to a narrow peak at $z_{1}$ with the width decreasing in time (Fig. (2)).

This describes the projection of the atomic quantum state to a final state with squeezed atom number at $K$ sites (a Fock states $\left|z_{1}, N-z_{1}\right\rangle$ with $z_{1}$ atoms at $K$ sites and $N-z_{1}$ atoms at $M-K$ sites). When $\delta z<1$, the final collapse is even faster than $\sqrt{\tau}$, due to the discreteness of $p(z, m, t)$. Measuring the photon number $m$ and time $t$, one can determine $z_{1}$ of a quantum trajectory.

In contrast to recent results in spin squeezing [16], which can be also obtained for thermal atoms [17], in our work, quantum nature of ultracold atoms is crucial, as we deal with the atom number fluctuations appearing due to the delocalization of ultracold atoms in space.

After the distribution shrinks to a single $z_{1}$, the light collapses to a single coherent state $\left|\alpha_{z_{1}}\right\rangle$, and the atoms and light get disentangled with a factorized state

$$
\left|\Psi_{c}\right\rangle=\left|z_{1}, N-z_{1}\right\rangle\left|\alpha_{z_{1}}\right\rangle .
$$

So, light statistics evolves from super-Poissonian to Poissonian. The conditioned (i.e., at a single trajectory) cavity photon number $\left\langle a_{1}^{\dagger} a_{1}\right\rangle_{c}(t)=|C|^{2} \sum_{z=0}^{N} z^{2} p(z, m, t)$ is given by the second moment of $p(z, m, t)$. Its dynamics [very similar to $\langle z\rangle_{c}$ in Fig. 2(c)] has jumps, even though all $\alpha_{z}(t)$ are continuous. In the no-count process, $\left\langle a_{1}^{\dagger} a_{1}\right\rangle_{c}$ decreases, while at one-count it jumps upwards, which is a signature of super-Poissonian statistics. Finally, it reduces to $\left\langle a_{1}^{\dagger} a_{1}\right\rangle_{c}=|C|^{2} z_{1}^{2}$, reflecting a direct correspondence between the final atom number and cavity photon number, which is useful for experiments.

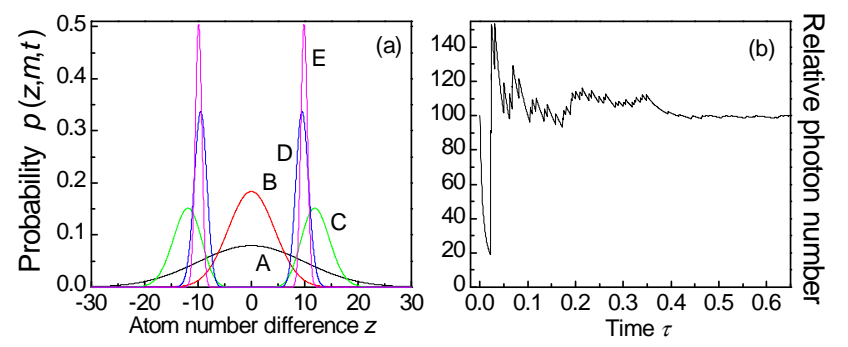

FIG. 3: (Color online) Photodetections at diffraction minimum. (a) Shrinking distribution of the atom-number difference for various times $\tau=0,0.02,0.03,0.17,0.65(\mathrm{~A}-\mathrm{E})$. The doublet corresponds to macroscopic superposition state. (b) Relative photon number $\left\langle a_{1}^{\dagger} a_{1}\right\rangle_{c} /|C|^{2}$ with quantum jumps. Initial state: $\mathrm{SF}, N=100$ atoms, $K=M=100$ sites.

Even the final Fock state still contains the atom-atom entanglement, as many components $\left|q_{1}, . ., q_{M}\right\rangle$ can have the same $z_{1}$. For example, the $\mathrm{SF}$ state can be represented as $|S F\rangle_{N, M}=\sum_{z} \sqrt{B_{z}}|S F\rangle_{z, K}|S F\rangle_{N-z, M-K}$ ( $B_{z}$ are binomial coefficients). After the measurement, it ends up in $|S F\rangle_{z_{1}, K}|S F\rangle_{N-z_{1}, M-K}$, i.e., the product of two uncorrelated superfluids.

Our measurement scheme determines (by squeezing) the atom number at a particular lattice region and projects the initial atomic state to some subspace. However, the atom number at different regions keeps quantum uncertainty. So, the quantum structure of the final state can be revealed in a further optical or matter-wave experiment. Thanks to the lattice geometry, one can change the illuminated region, and further study measurementinduced collapse of the state in the remaining subspace.

Even in matter-wave experiments [1], the product of SFs will look different from the initial SF: the atoms from different regions will not interfere in average. Note, that we did not specify how $K$ sites were selected. One can illuminate a continuous region. However, one can illuminate each second site by choosing the probe wavelength twice as lattice period and get number squeezing at odd and even sites. In this way, one gets a measurementprepared product of two SFs "loaded" at sites one by one (e.g. atoms at odd sites belong to one SF, while at even sites to another). While the initial SF shows the long-range coherence $\left\langle b_{i}^{\dagger} b_{j}\right\rangle$ with the lattice period, the prepared state will demonstrate the doubled period in $\left\langle b_{i}^{\dagger} b_{j}\right\rangle$ ( $b_{j}$ is the atom annihilation operator).

Transverse probing at diffraction minimum. In contrast to classical atoms, quantum gases scatter light even in diffraction minima [3]. Here $z=D_{10}^{q}=$ $\sum_{j=1}^{M}(-1)^{j+1} q_{j}$ is the atom number difference between odd and even sites, varying from $-N$ to $N$ with a step 2 (we assumed $K=M$ ). Eq. (77) keeps its form with a new meaning of $z$ and $p_{0}(z)$ [for $\mathrm{SF}$, new $p_{0}(z)$ is nearly a Gaussian centered at $z=0$ and the width $\sqrt{N}[3]$ ].

The striking difference from the diffraction maximum 
is that our measurement (7) is not sensitive to the sign of $z$, while the amplitudes $\alpha_{z}=C z$ are. So, the final state is a macroscopic superpositions of two Fock states with $z_{1,2}= \pm \sqrt{m / \tau}$ and light amplitudes: $\alpha_{z_{2}}=-\alpha_{z_{1}}$,

$$
\left|\Psi_{c}\right\rangle=\left(\left|z_{1}\right\rangle\left|\alpha_{z_{1}}\right\rangle+(-1)^{m}\left|-z_{1}\right\rangle\left|-\alpha_{z_{1}}\right\rangle\right) / \sqrt{2} .
$$

Figure 3 shows the collapse to a doublet probability $p\left( \pm z_{1}, m, t\right)$ and the photon-number trajectory, where upward jumps and no-count decreases can be seen.

In contrast to a maximum, even in the final state, the light and matter are not disentangled. Moreover, to keep the purity of the state, one should know precisely the number of detected photons, because of the sign flip in Eq. (9). This reflects the fragility of macroscopic superposition states with respect to the decoherence.

As a result, the measurement-based state preparation at the diffraction maximum (8) is much more robust.

For probing through a mirror $\left(\eta \neq 0, a_{0}=0\right)$, in contrast to the transverse probing, the probability distribution can collapse both to a singlet and doublet. Importantly, here the superposition state can be more robust than (9). This is due to a smaller phase jump between two Fock states, which can be obtained from Eqs. (3)-(5).

Deviations from our ideal setup can slightly modify the results, i.e., the mode profiles will lead, instead of atom number, to more general variables given by $\hat{D}_{l m}$.

In summary, we showed that using the light-matter entanglement in ultracold gases enables QND measurements of different atomic variables and creation of specific atomic states. The state type is determined by the optical geometry. Our model can be generalized to other quantum arrays. Cavity QED with quantum gases can operate with atom numbers from millions to one 18. Thanks to recent experimental breakthroughs [2], preparing various kinds of atom number squeezing is already doable, and creation of the superposition states with, at least, small particle number [10] may become practical.

We thank D. Ivanov, E. Polzik, and A. Vukics for stimulating discussions. Support by FWF (P17709, S1512).
* Electronic address: Igor.Mekhov@uibk.ac.at

[1] D. Jaksch et al., Phys. Rev. Lett. 81, 3108 (1998); I. Bloch, Nature Phys. 1, 23 (2005); M. Lewenstein et al., Adv. Phys. 56, 243 (2007).

[2] F. Brennecke et al., Nature 450, 268 (2007); Y. Colombe et al., Nature 450, 272 (2007); S. Slama et al., Phys. Rev. Lett. 98, 053603 (2007); I. Teper, Y. J. Lin, and V. Vuletic, Phys. Rev. Lett. 97, 023002 (2006).

[3] I. B. Mekhov, C. Maschler, and H. Ritsch, Nature Phys. 3, 319 (2007); Phys. Rev. Lett. 98, 100402 (2007); Phys. Rev. A 76, 053618 (2007).

[4] W. Chen, D. Meiser, and P. Meystre, Phys. Rev. A 75, 023812 (2007).

[5] C. Maschler, I. B. Mekhov, and H. Ritsch, Eur. Phys. J. D 146, 545 (2008); A. Vukics, C. Maschler, and H. Ritsch, New J. Phys. 9, 255 (2007); C. Maschler, and H. Ritsch, Phys. Rev. Lett. 95, 260401 (2005); J. Larson et al., Phys. Rev. Lett. 100, 050401 (2008).

[6] C. Orzel et al., Science 291, 2386 (2001).

[7] T. Volz et al., Nature Phys. 2, 692 (2006).

[8] J. Kasprzak et al., Nature 443, 409 (2006).

[9] J. Gambetta et al., Phys. Rev. A 77, 012112 (2008).

[10] A. Ourjoumtsev et al., Science 312, 83 (2006); J. S. Neergaard-Nielsen et al., Phys. Rev. Lett. 97, 083604 (2006); M. Brune et al., Phys. Rev. Lett. 77, 4887 (1996).

[11] M. Brune et al., Phys. Rev. A 45, 5193 (1992).

[12] Y. P. Huang and M. G. Moore, Phys. Rev. A 73, 023606 (2006); J. Ruostekoski et al., Phys. Rev. A 57, 511 (1998); J. F. Corney and G. J. Milburn, Phys. Rev. A 58, 2399 (1998); D. A. R. Dalvit, J. Dziarmaga, and R. Onofrio, Phys. Rev. A 65, 033620 (2002).

[13] W. M. Itano et al., Phys. Rev. A 47, 3554 (1993).

[14] H. Carmichael, An Open System Approach to Quantum Optics (Springer, Berlin, 1993).

[15] T. Yu. Ivanova and D. A. Ivanov, Opt. Commun. 272, 148 (2007); C. W. Gardiner and P. Zoller, Quantum Noise (Springer, Berlin, 1999).

[16] K. Eckert et al., Nature Phys. 4, 50 (2008).

[17] J. Hald et al., Phys. Rev. Lett. 83, 1319 (1999); D. Meiser, J. Ye, and M. J. Holland, arXiv:0707.3834.

[18] P. Maunz et al., Nature 428, 50 (2004). 